Recepción: 23 / 09 / 2018

Aceptación: 17 / 11 / 2018

Publicación: 03 / 12 / 2018

Ciencias económicas y empresariales

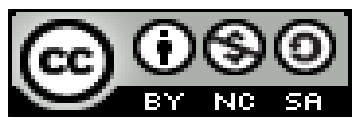

Artículo original

\title{
Sistema de control de préstamos de activos electrónicos para la biblioteca central de la Universidad Técnica de Manabí
}
Control system for electronic asset loans for the central library of the Technical University of Manabi

\section{Sistema de controle de empréstimos de ativos eletrônicos para a biblioteca central da Universidade Técnica de Manabí}

\author{
Jenmer M. Pinargote-Ortega ${ }^{\mathrm{I}}$ \\ maricela.pinargote@fci.edu.ec \\ Gabriel P. Demera-Ureta II \\ gabriel.demera@fci.edu.ec \\ Marely del R. Cruz-Felipe ${ }^{\text {III }}$ \\ marely.cruz@fci.edu.ec \\ Mérida M. Moreira-Moreira ${ }^{\mathrm{IV}}$ \\ m4_90@hotmail.com
}

Correspondencia: maricela.pinargote@fci.edu.ec

\footnotetext{
${ }^{\text {I }}$ Magíster en Pedagogía, Ingeniera en Sistemas Informáticos, Docente de la Universidad Técnica de Manabí, Portoviejo, Manabí, Ecuador.

II Magíster en Docencia e Investigación Educativa, Ingeniero en Sistemas Computacionales, Docente de la Universidad Técnica de Manabí, Portoviejo, Manabí, Ecuador.

III Doctora en Ciencias Técnicas, Ingeniera en Equipos y Componentes Electrónicos, Docente de la Universidad Técnica de Manabí, Portoviejo, Manabí, Ecuador.

IV Ingeniera en Sistemas Informáticos, Docente de la Universidad Técnica de Manabí, Portoviejo, Manabí, Ecuador.
} 


\section{Resumen}

La presente investigación se realizó con la finalidad de contribuir al mejoramiento de una atención personalizada y de calidad en la biblioteca central de la Universidad Técnica de Manabí; en la que se aplicó el método bibliográfico e investigación de campo, otorgándole un carácter científico-racional a la información obtenida con técnicas de entrevista al personal bibliotecario y fichas de observación, lo que permitió demostrar que los procesos de control de préstamos de activos electrónicos se realizan de manera manual, acarreando pérdida o deterioro de información; por tal razón se diseñó, desarrolló e implementó un sistema de control de préstamos de activos electrónicos tales como laptops y tablets, los resultados a los que se llegó fue simplificar las labores del bibliotecario, registrar: detalles del préstamo con tiempo de inicio y finalización, personas que intervienen tanto en la emisión, recepción y finalización del préstamo; se concluyó que todos estos procedimientos son de esencial importancia para el registro, comprobación e inspección en cualquier momento del uso y calidad del servicio que brinda la biblioteca central con el préstamo de los activos electrónicos; siendo los principales beneficiarios estudiantes, personal administrativos y docentes de la Universidad Técnica de Manabí, diversas instituciones educativas, y la comunidad en general, comprometidos con el avance tecnológico, educativo y cultural.

Palabras clave: activos electrónicos; biblioteca central; calidad del servicio; sistema de control de préstamos; Universidad Técnica de Manabí.

\section{Abstract}

This investigation was made with the goal of contributing to a better and personalized service at the Universidad Técnica de Manabí central library. The methods used were bibliographic research and field research, obtaining a rational-scientific view of the information obtained with interview techniques towards the library personal and observation charts. These studies demonstrate that the electronic item loan process is done manually, inducing loss or detriment to the information; for this reason a system was designed to control to electronic items such as laptops and tablets. The results obtained simplified the librarians' task, registering details of the loan such as time as start and end of loan, people who intervene in the emission, reception and finalization of the loan, as well as theft attempts of said devices and their respective reports. We conclude that all these procedures are of essential importance to the registration, verification and 
inspection at any point during the loan, and service quality that the Central Library provides with the loan of electronics devices, the main beneficiaries being the students, administrative personal and teachers at the Universidad Técnica de Manabí, several educational institutions at the community in general, focused on the technological, educational and cultural advancement.

Keywords: electronic assets; central library; quality of service; loan control system; Technical University of Manabí.

\section{Resumo}

A presente investigação foi realizada com o objetivo de contribuir para a melhoria da atenção personalizada e da qualidade na biblioteca central da Universidade Técnica de Manabí; no qual o método bibliográfico e a pesquisa de campo foram aplicados, concedendo um caráter científicoracional à informação obtida com as técnicas de entrevista para o pessoal bibliotecário e as folhas de observação, o que permitiu demonstrar que os processos de controle de empréstimos de ativos eletrônicos realizar manualmente levando a perda ou deterioração da informação; Por esse motivo, foi concebido, desenvolvido e implementado um sistema de controle de empréstimos para ativos eletrônicos, como laptops e tablets. Os resultados obtidos foram para simplificar o trabalho do bibliotecário, registro: detalhes do empréstimo com horário de início e término, pessoas que intervêm na emissão, recepção e rescisão do empréstimo; concluiu-se que todos esses procedimentos são de importância essencial para o registro, verificação e inspeção em qualquer momento do uso e qualidade do serviço prestado pela Biblioteca Central com o empréstimo dos ativos eletrônicos; sendo os principais beneficiários estudantes, funcionários administrativos e docentes da Universidade Técnica de Manabi, várias instituições educacionais e a comunidade em geral comprometida com o avanço tecnológico, educacional e cultural.

Palavras chave: ativos eletrônicos; biblioteca central; qualidade do serviço; sistema de controle de empréstimos; Universidade Técnica de Manabí.

\section{Introducción}

Los sistemas automatizados de biblioteca, son un conjunto organizado de recursos humanos que utilizan dispositivos y programas informáticos (Díaz, 2013), adecuados a la naturaleza de los datos que deben procesar, para realizar procesos y facilitar los servicios que permiten alcanzar los objetivos de la biblioteca: almacenar de forma organizada el conocimiento humano contenido en 
todo tipo de materiales bibliográficos para satisfacer la necesidades informativas, recreativas y de investigación de los usuarios (García, 1999).

Desde el punto de vista histórico, el interés de la automatización bibliotecaria se ha centrado en operaciones internas no visibles inmediatamente para el usuario (Pérez, 1996). Sin embargo, el objetivo de tales esfuerzos ha sido mejorar la calidad de los servicios ofrecidos al público (Navarrete, 2014).

La automatización hace muchos años dejó de ser una moda y se convirtió en una necesidad para las bibliotecas. La tecnología se transformó en una herramienta indispensable para el buen desempeño de los procesos y servicios, así como para el intercambio de información con otras bibliotecas (Navarrete, 2008) (Navarrete, 2014).

La automatización de los catálogos, junto con la del préstamo a través de la base de datos de usuarios, facilita tareas de evaluación del uso, de la circulación de materiales, así como el control de calidad (Gómez, 2002) (Fushimi, 2011).

La biblioteca central de la Universidad Técnica de Manabí (UTM), nace como una necesidad de renovar y ampliar el acervo bibliográfico inculcando el apoyo y la orientación con las actividades propias de la docencia y del proceso enseñanza-aprendizaje.

Con las exigencias de la educación actual se emprendió un programa de mejoras, adecuaciones y ampliación de su cobertura de servicios en el área tecnológica en la biblioteca central-UTM, conforme avanzan los días la demanda incrementa y al ser un procedimiento manual se ven aglomeraciones de estudiantes para hacer uso del activo electrónico o para retirar sus identificaciones al momento de abandonar las instalaciones, el procedimiento debe repetirse cada vez que el estudiante solicite el préstamo de un activo electrónico, provocando contrariedad en los involucrados del préstamo; siendo también una carga para el bibliotecario en cuanto al control de la integridad de los activos electrónicos.

Por tales razones, se implementó un sistema de control de préstamos para los activos electrónicos (tablet y laptops), lo cual contribuye que el bibliotecario brinde una atención personalizada y de mejor calidad a la comunidad universitaria y general que soliciten el servicio de préstamo de 
activos electrónicos; ahorrando tiempo y esfuerzos, a la vez que se ligan responsabilidades entre los actores del préstamo.

La investigación fue factible gracias a la información confiable que proporcionaron los administrativos de la biblioteca central-UTM, en donde los principales beneficiaros son los estudiantes que tienen a su alcance las herramientas tecnológicas requeridas para su proceso de aprendizaje, los docentes se ven favorecido en su método de enseñanza con el uso de la tecnología y además las autoridades de la biblioteca central-UTM que tienen a su disposición un sistema informático para administrar y controlar el uso correcto de sus activos electrónicos.

Es de gran importancia porque favorece los procesos pedagógicos permitiendo que los estudiantes encuentren en las tecnologías informáticas el apoyo que necesitan para su formación académica y los responsables del préstamo de los activos electrónicos, pueden contar con un software a medida para el registro de los préstamos.

\section{Metodología}

Se utilizó los siguientes métodos:

- Bibliográfico: mediante este método, se recopiló información necesaria para fundamentar de manera científica las tecnologías, lenguajes de programación, herramientas y modelo de proceso a implementar.

- Investigación de campo: con este método, se logró comprobar la veracidad y certeza de los problemas que producía el sistema manual de préstamos de los activos electrónicos en la biblioteca central-UTM.

- Descriptiva: permitió describir el problema y la solución que se suscita en la biblioteca central-UTM.

- Propositiva: se dio solución a la problemática planteada, implementando un sistema informático para el control de préstamos de activos electrónicos.

- Observación: por medio de esta técnica se recopiló los procesos de registro y control de los activos electrónicos de forma manual. 
- Entrevista: permitió recopilar requerimientos en forma verbal, a través de formularios de preguntas previamente elaboradas. Se entrevistó a los administrativos de la biblioteca centralUTM.

\section{Resultados}

En la solución, se incorporaron modelos de proceso, lenguajes de programación, herramientas y tecnologías para satisfacer las necesidades de la biblioteca central-UTM.

\section{Selección de modelo de proceso, lenguajes de programación, herramientas y tecnologías}

Metodología ágil SCRUM: se aplicó por adoptar una estrategia de desarrollo iterativa (Pressman et al., 2015) (Martel, 2014), se efectuó en corto tiempo la iteración del sistema de control de préstamo capturando requerimientos prioritarios, permitiendo a futuro implementar nuevas iteraciones.

A través de la entrevista a la directora de la biblioteca central-UTM y con el uso de fichas de observación, documentación se identificó y se analizó requerimientos de los procesos manuales que se llevan durante el préstamo de los activos electrónicos, lo cual ayudó para el diseño de la base de datos y el desarrollo del sistema de control de activos electrónicos (laptops y tablets).

- Shardevelop: la aplicación incorpora sus propios analizadores sintácticos (Russell et al., 2012). Se utilizó por ser un entorno de desarrollo integrado libre para lenguajes de programación C\#, Visual Basic .NET, F\#, Python, Ruby, Boo, C++, entre otros (Stolfa et al., 2017).

- MySQL administrator: herramienta que permite realizar tareas administrativas sobre servidores de MySQL (Cabral et al., 2011) (DuBois, 2013) (Cote et al., 2017). Mismo que permitió administrar la base de datos "prestamo-equipos".

- MySQL query browser: soporta vistas, procedimientos almacenados y funciones almacenadas (Bell, 2010) (Aristizábal, 2012). Permitió de una manera más intuitiva y gráfica la consulta y la edición de datos.

- PLSQL developer: es un entorno de desarrollo integrado que está específicamente dirigido al desarrollo de unidades de programas almacenados para bases de datos Oracle (Odewahn, 
2000) (Gupta, 2016). Se pudo comparar unidades de programa, tablas, vistas, secuencias con fuentes en la base de datos para la importación de registros de estudiantes matriculados.

- MySQL server: sistema de gestión de bases de datos relacional, multihilo y multiusuario con un esquema de licenciamiento dual (Thibaud, 2006) (DuBois, 2013). Permitió administrar la base de dato "prestamo-equipos".

- Sistemas de identificación automática: los sistemas de código de barras, están compuestos por una serie de barras negras y blancas (Monteros, 2014), de manera vertical y una serie de números inferiores, este código permite la identificación gracias al uso de lectores laser o pistolas de código de barra. La versión puede varias según el país (Bustio, 1993) (Torres, 2014).

Se analizaron algunas tecnologías para el sistema de código de barras como: Codabar, en el sector médico, el código 2/5 utilizado en la industria del automóvil, contenedores de barcos, industria pesada en general o el código 39, usado en procesos industriales, logísticos o librerías (Cooper et al., 2016).

Se implementó la codificación de barras para el préstamo de los activos electrónicos, usando la codificación EAN-13 (Torres, 2014), se etiquetó laptops y tablets, mismas que son emitidas y recibidas en préstamo escaneando el código de barra asociado al activo electrónico en cuestión.

Se utiliza para el escaneo el lector SG10T, equipo plug-and-play que se conecta mediante interfaz USB o emulación de teclado (Monteros, 2016), cimentado en la tecnología de imagen, lee de forma sencilla el código de barra ya que cuenta con un haz de luz que facilita la tarea de apuntar al código (Campo, 2016), sin necesidad de una formación específica al usuario.

El sistema de control (Morales et al., 2013), compara la lectura del código de barra con los datos almacenados en la base de datos para realizar el préstamo de los activos electrónicos.

\section{Modelo de base de datos}

En la base de datos se almacena los datos de: usuarios que tienen acceso al sistema, personas que realizan préstamos, equipos electrónicos, préstamos y datos de operaciones ejecutadas que posteriormente pueden ser auditadas. 


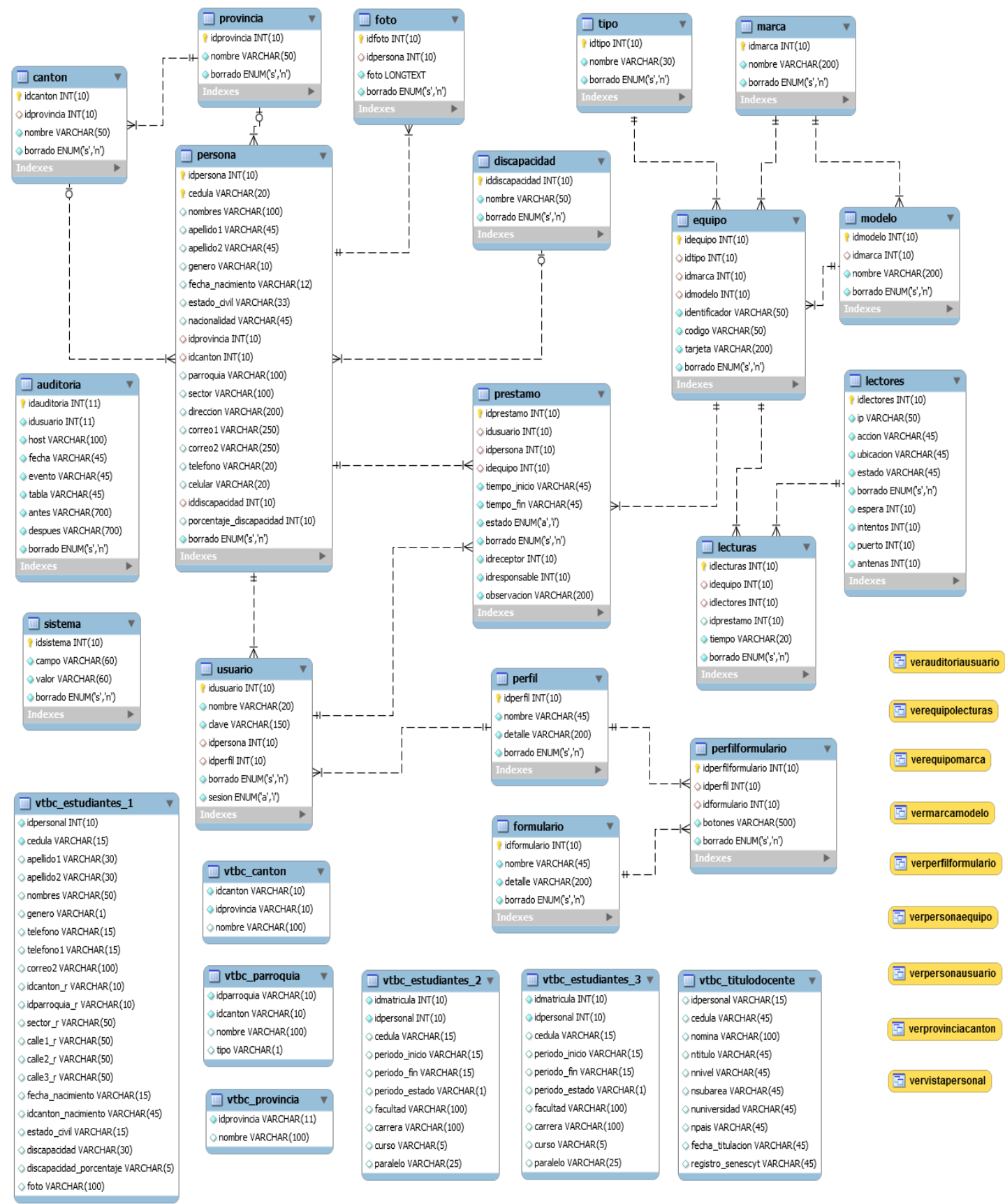

Figura 1: modelo de base de datos

Fuente: elaboración propia

\section{Sistema de control de préstamo}

Se implementó un sistema de escritorio que se conecta a un servidor de base de datos, mismo que permite consulta, ingreso, modificación y eliminación de registros de información; se puede 
respaldar y restaurar la base de datos desde el mismo sistema, así como administrar las operaciones que realiza el usuario y realizar alguna auditoria si se considera necesario.

Las funcionalidades principales se detallan a continuación:

\section{- Acceso al sistema}

Se realiza logueo, especificando un nombre de usuario y una clave de acceso proporcionados por el administrador del sistema.

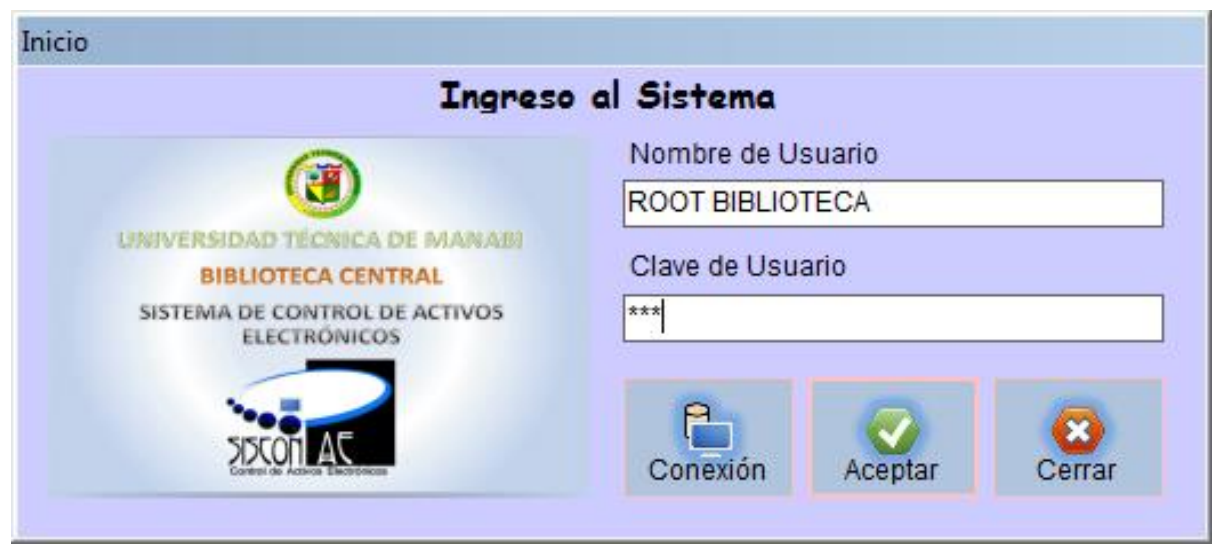

Figura 2: pantalla de acceso al sistema

Fuente: elaboración propia

\section{- Pantalla principal}

Una vez que se ha iniciado sesión, aparece una pantalla de bienvenida donde están disponibles todas las opciones del sistema, algunas de las cuales estarán o no habilitadas de acuerdo a los permisos definidos para cada tipo de usuario.

1. Menú de opciones del sistema en forma de menú desplegable.

2. Opciones de acceso rápido a las principales opciones del menú desplegable.

3. La interfaz muestra tipo de usuario y el usuario que ha ingresado al sistema, así como la fecha y hora actual del servidor de datos.

4. Ventanas abiertas a través de los botones de acceso rápido o el menú desplegable correspondiente. 


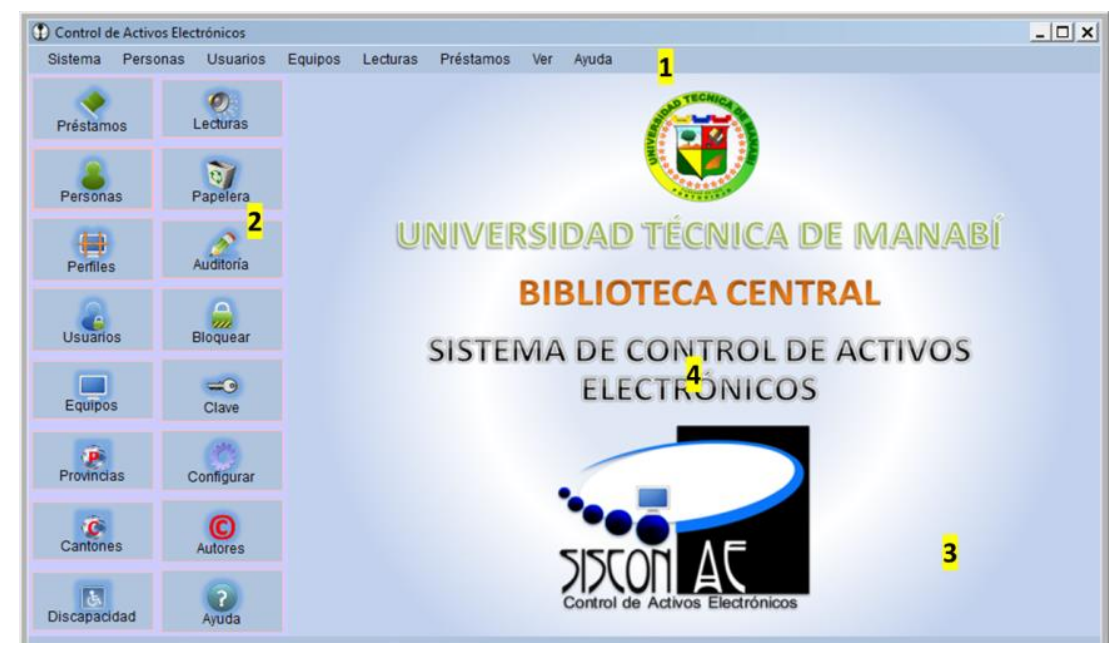

Figura 3: menú de opciones en la pantalla principal

Fuente: elaboración propia

\section{- Actualización de datos}

El sistema permite actualizar los datos personales y la información académica de los estudiantes o docentes de la Universidad Técnica de Manabí, para ello se le solicita al centro de cómputo de la UTM un archivo CSV con los últimos registros de estudiantes matriculados y los datos respectivos de dicha matrícula.

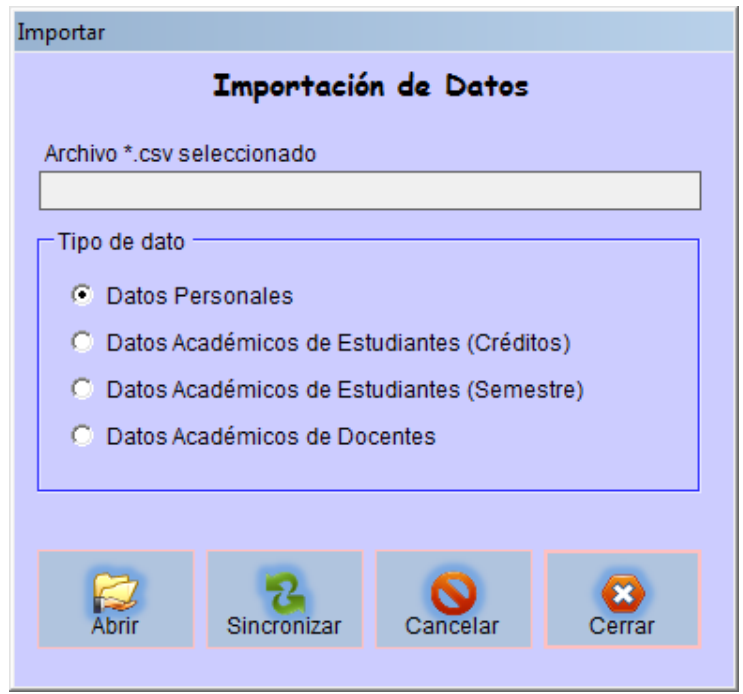

Figura 4: importación de datos

Fuente: elaboración propia 
Para el ingreso y edición de información se utiliza una interfaz, donde se debe especificar los datos necesarios para completar el proceso de ingreso o actualización de datos.

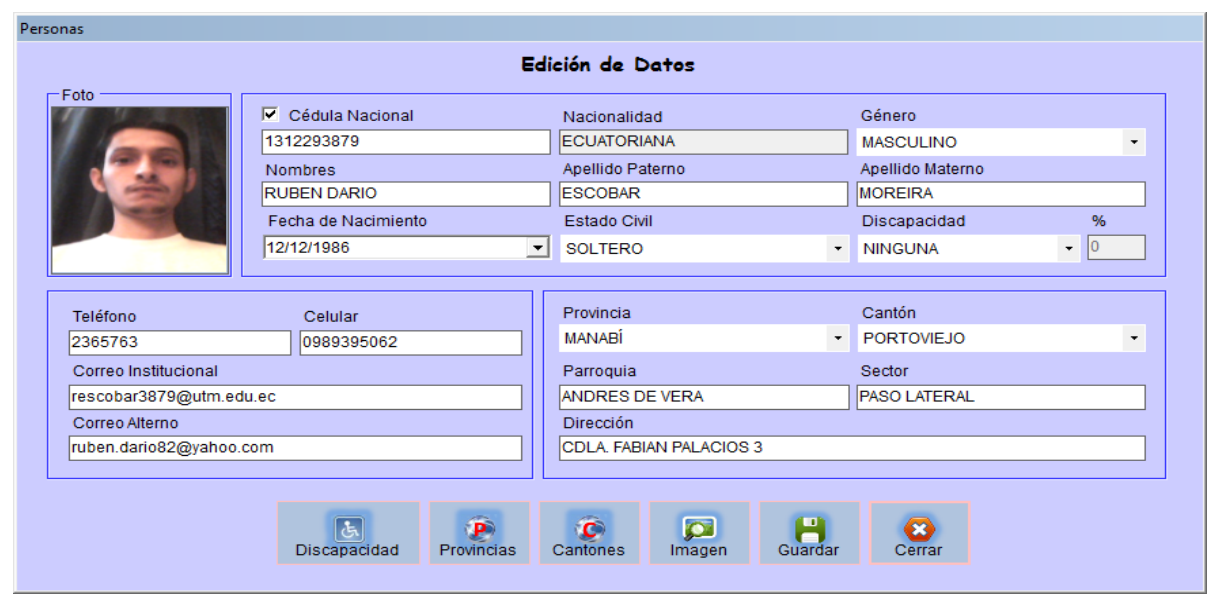

Figura 5: edición de datos

Fuente: elaboración propia

\section{- Registro de equipos}

La aplicación permite procesar la información de los equipos que se va a utilizar con el identificador, código de barra, tipo, marca y modelo.

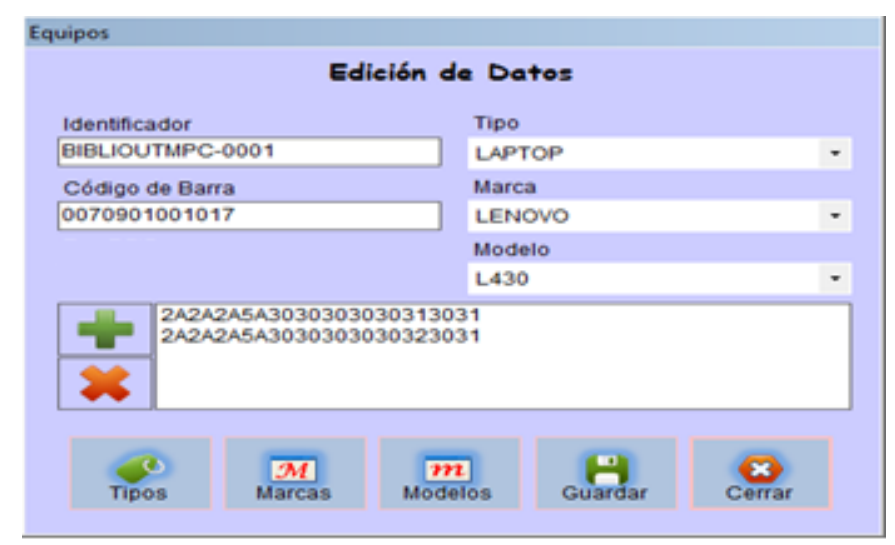

Figura 6: registro de equipos

Fuente: elaboración propia

\section{- Préstamos equipos}

Para ejecutar el préstamo se elige al prestamista y al activo electrónico por identificador del equipo o por código de barra. 


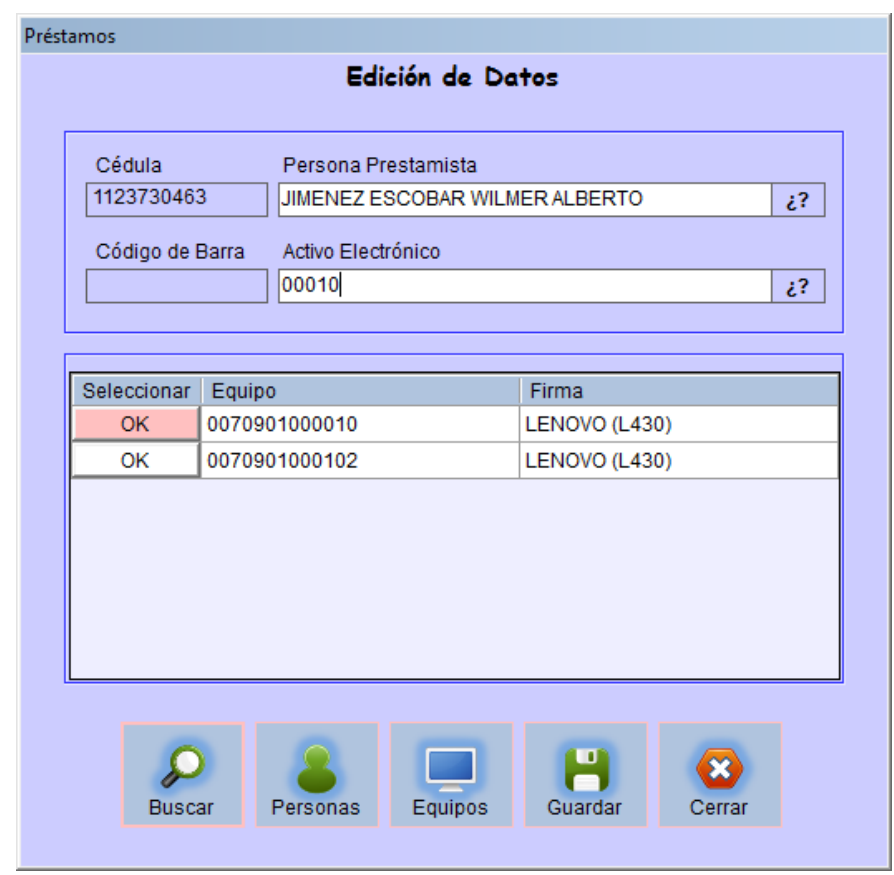

Figura 7: préstamos de equipo

Fuente: elaboración propia

Para la finalización del préstamo, el código de barra del equipo debe ser leído por el escáner, no se admite copia o ingreso de datos para asegurar que el préstamo se está finalizando correctamente.

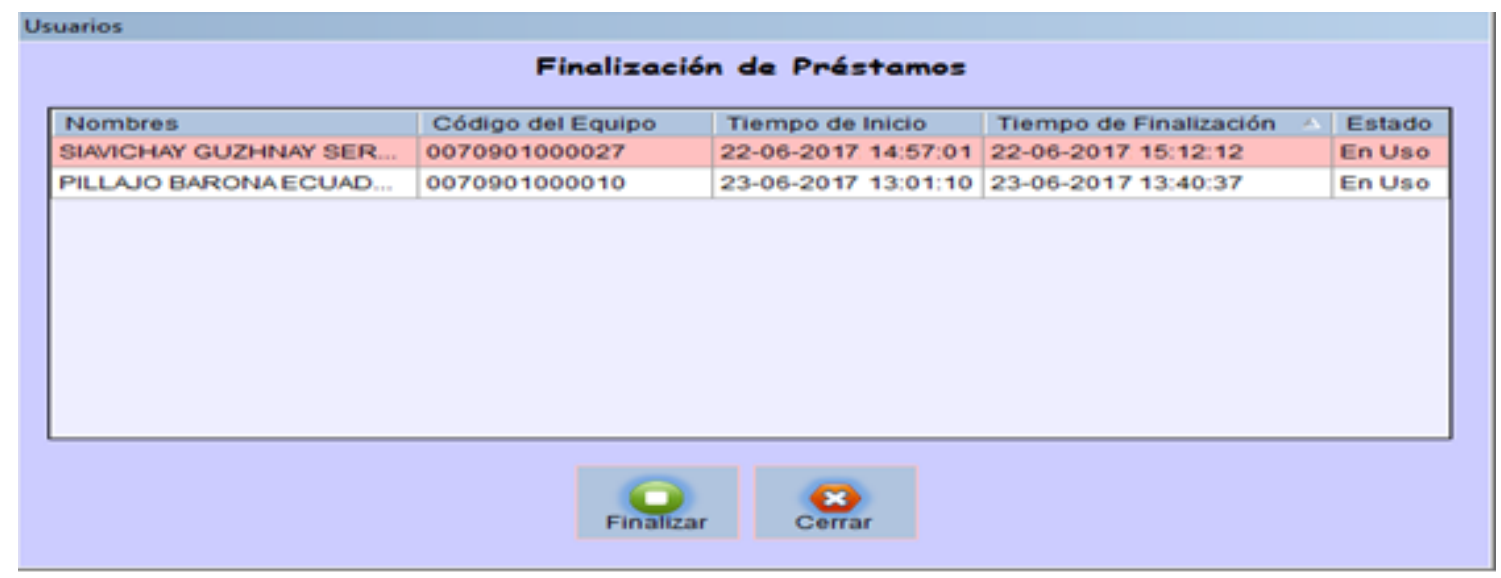

Figura 8: finalización de préstamos

Fuente: elaboración propia 


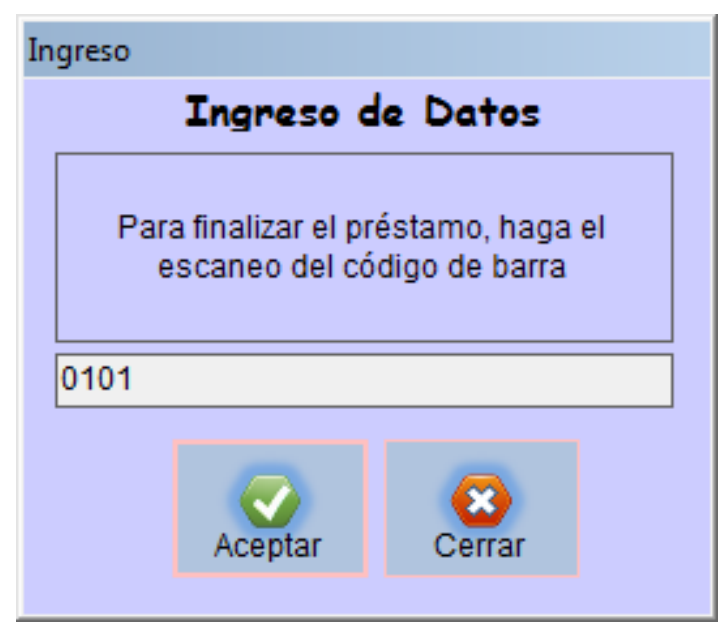

Figura 9: lectura del código de barra para finalización del préstamo

Fuente: elaboración propia

\section{- Transferencia de responsabilidad}

Si el usuario actualmente logueado ha realizado algún préstamo, se le impedirá bloquear o finalizar su sesión hasta que termine los préstamos que realizó o transfiera esta responsabilidad a otro usuario con los permisos adecuados.

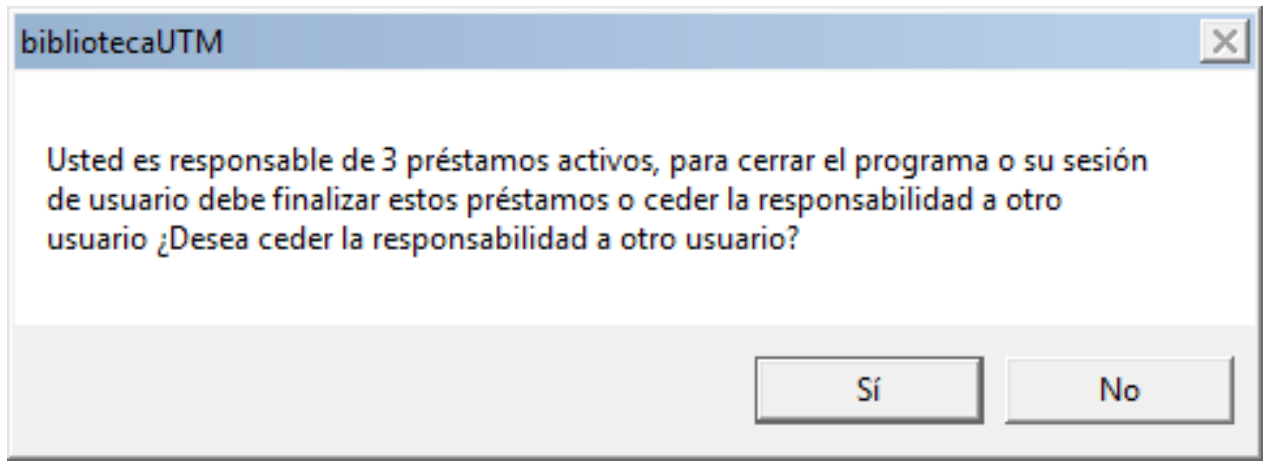

Figura 10: finalización de responsabilidad

Fuente: elaboración propia

En el caso de querer transferir la responsabilidad, el usuario que asumirá la terminación de estos préstamos deberá proporcionar sus datos de autenticación, si son válidos se mostrará un mensaje indicando que se han asumido los préstamos y automáticamente se finalizará la sesión del usuario actual y se activará la sesión del usuario que asumió la responsabilidad. 


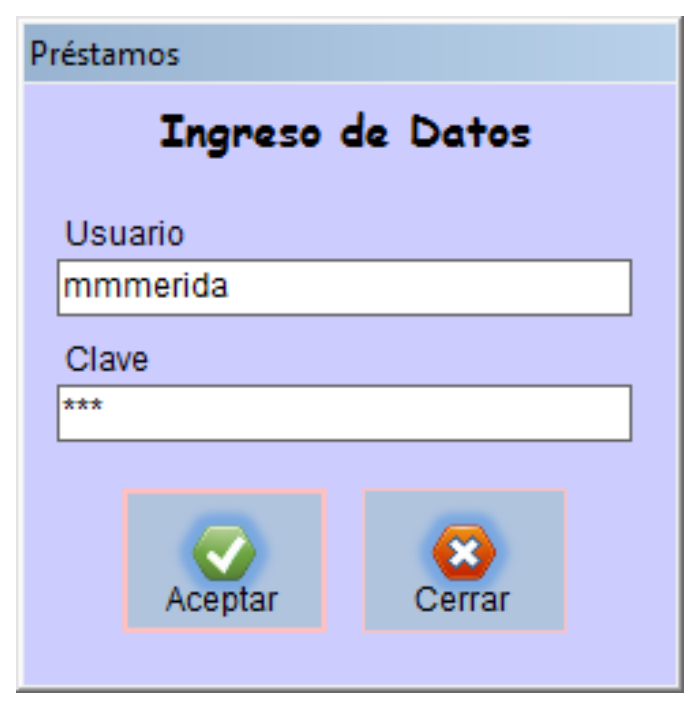

Figura 11: transferencia de responsabilidad

Fuente: elaboración propia

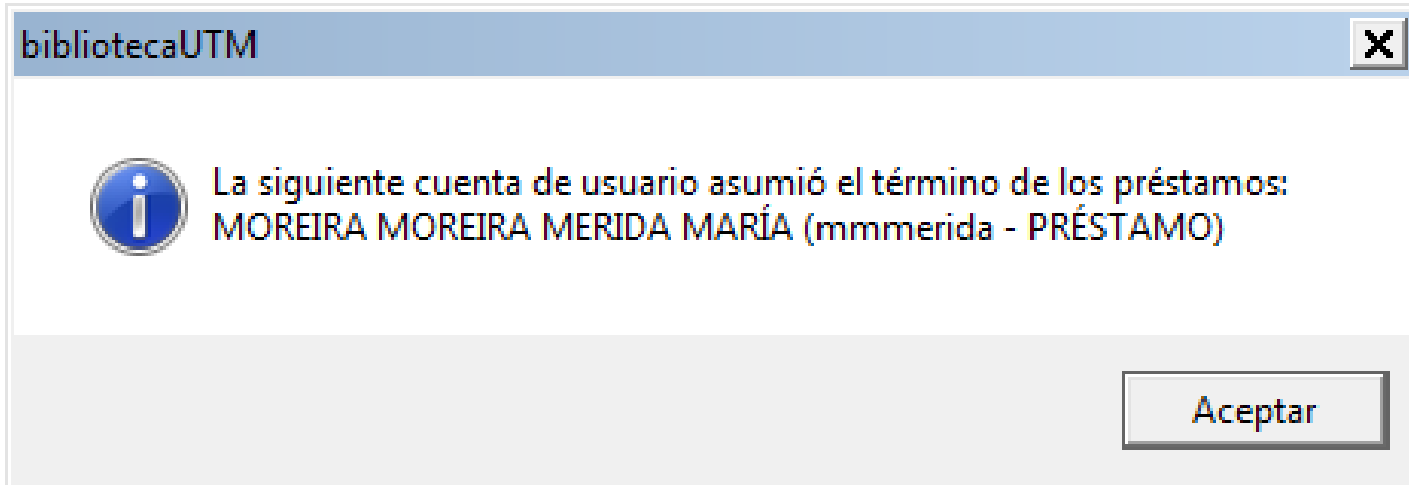

Figura 12: nueva cuenta activa

Fuente: elaboración propia

\section{- Auditoría}

Se procesa la información de los registros de auditoría que se almacenan cuando el usuario opera sobre la información contenida en la base de datos del sistema a través de las distintas interfaces del mismo.

El respaldo de la tabla de auditorías y el vaciado respectivo de la misma se hace automáticamente cada vez que se alcanzan los 500 registros, pero también se pueden hacer respaldos manuales dando clic en el botón "Respaldo". Se puede auditar escogiendo el respaldo que se necesite. 


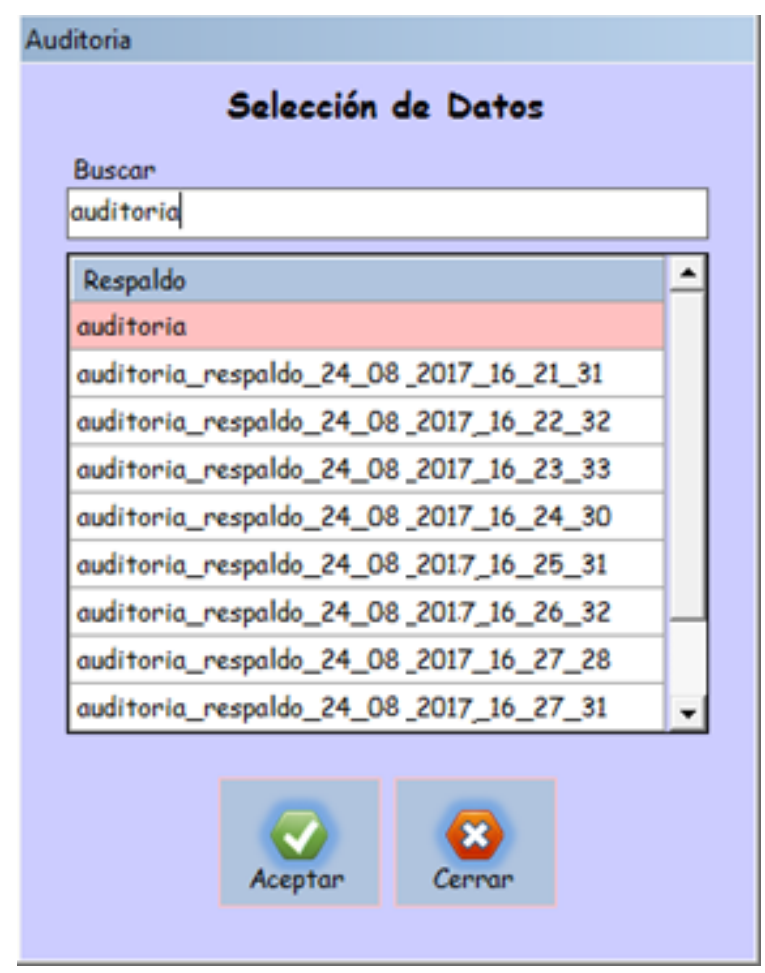

Figura 13: respaldo de auditoria

Fuente: elaboración propia

Al seleccionar se muestra información específica del registro que se desee auditar.

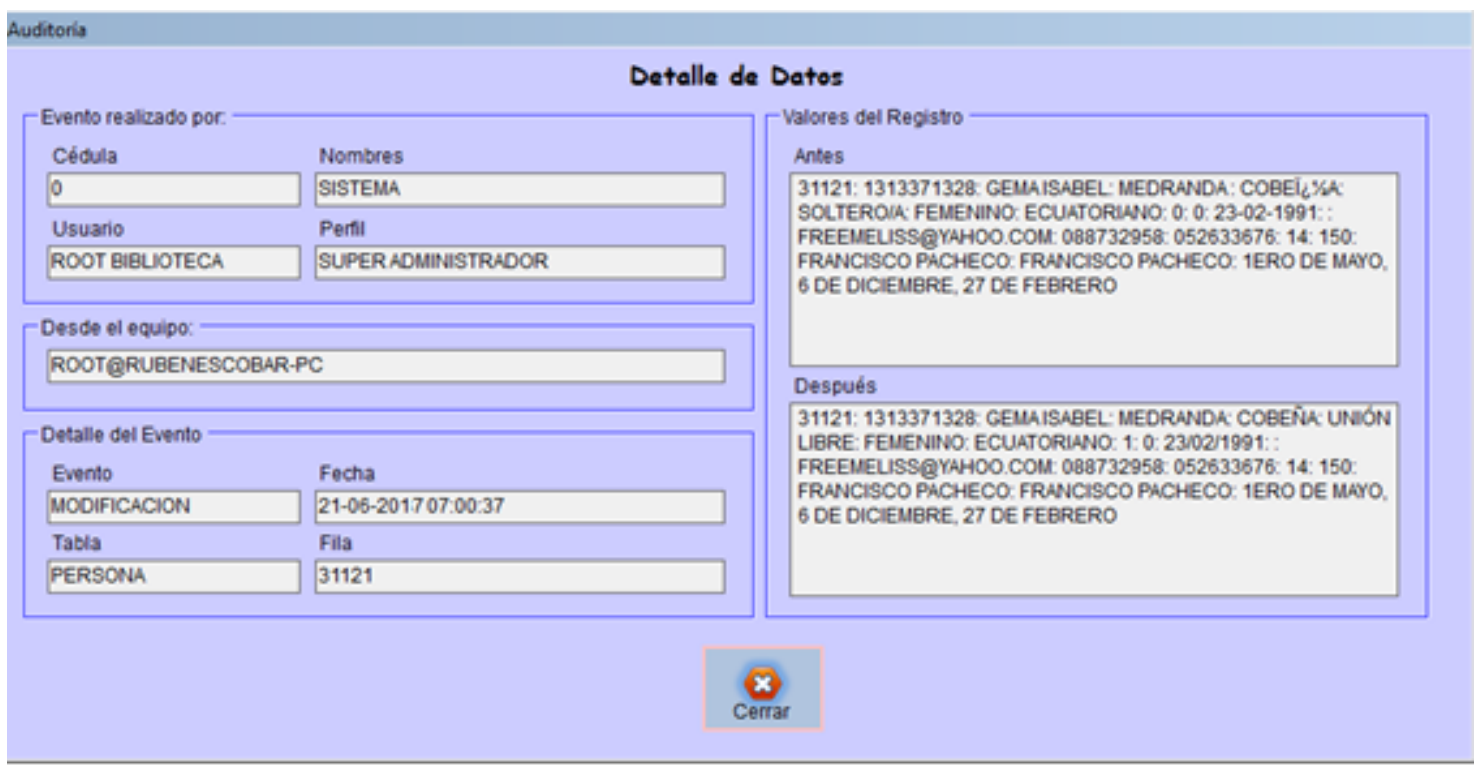

Figura 14: detalle de auditoria

Fuente: elaboración propia 


\section{Conclusiones}

El sistema de control de préstamos que se implementó en la biblioteca central-UTM se sustenta con el uso de herramientas como: Sharpdevelop que se caracteriza por ser un entorno de desarrollo integrado libre, MySql que permite velocidad y flexibilidad en las transacciones que se realizan en una base de datos relacional y la tecnología de identificación automática que es una alternativa eficiente para mejorar los procesos de control de activos electrónico, lo que permitirá realizar futuros incrementos del sistema.

Con la implementación del sistema informático se brindará una atención adecuada con un respectivo control en los préstamos realizados de los activos electrónicos (tablets y laptops), y se obtendrá reportes de manera rápida y eficaz, ligando responsabilidades entre los actores del préstamo.

Además, contribuye que el personal bibliotecario brinde una atención personalizada y de calidad prestando los activos electrónicos de forma segura y confiable a la comunidad universitaria y general para que potencien y acrecienten nuevos conocimientos.

Gracias al servicio de préstamo de activos electrónicos, se fortalecerá los procesos pedagógicos y se incentivará el interés en la investigación científica para el desarrollo de la provincia y del país, permitiendo que la comunidad universitaria y general encuentren en las tecnologías informáticas el apoyo que necesitan para su formación académica.

\section{Referencias Bibliográficas}

Aristizábal, D. (2012). PHP básico y práctico. Colombia.

Bell, C., Kindahl, M., Thalmann, L. (2010). MySQL High Availability: Tools for Building Robust Data Centers. United States of America.

Bustio, J. (1993). Sistema de identificación y control automático (II). España.

Cabral, S. y Murphy K. (2011). MySQL adminstrator's. Indiana.

Campo, A. (2016). UF0035-Operaciones de caja en la venta. España.

Cooper, D., Schindler, P. (2016), Métodos de pesquisa em Administração. Brasil. 
Cote, C., Lah, M., Sarka, D. (2017). SQL Server 2017 Integration Services Cookbook. Reino Unido.

Díaz, D. (2013). Bibliotecas públicas, nuevas tecnologías de la información: impacto en el personal bibliotecario. EEUU.

DuBois, P. (2013). MySQL. España.

Equipo de Código de Barras y Auto id - Tecnología de identificación automática y punto de venta, Equipo de Código de Barras y Auto id. Recuperado el 12 de diciembre del 2016 de: http://www.idautomatica.com.

Fushimi, M. (2011). Evaluación de bibliotecas universitarias: una propuesta desde las perspectivas objetiva y subjetiva. Buenos aires.

García, M. (1999). Automatización de bibliotecas. Madrid: Arco/Libros.

Gómez, J. (2002). Gestión de bibliotecas. Universidad de Murcia. España.

Gupta, S. (2016). Advanced oracle PL/SQL developer's guide. Reino Unido.

Martel, A. (2014). Gestión práctica de proyectos con SCRUM: desarrollo de software ágil para el SCRUM master. España.

Monteros, I. (2014). Montaje y mantenimiento de sistemas y componentes informáticos. España.

Monteros, I. (2016). Equipos microinformáticos. España.

Morales, R., Ramírez, R. (2013). Sistemas de control moderno. Volumen I: sistemas de tiempo continúo. México.

Navarrete, O. (2014). Bibliotecas universitarias y automatización. México.

Navarrete, O. y Butrón, K. (2008). Sistemas integrales para la automatización de bibliotecas basados en software libre. ACIMED. 18(6).

Odewahn, A. (2000). Oracle Web Applications: PL/SQL Developer's Introduction. United States of America. 
Pérez, A. (1996). Perfil y nivel de satisfacción de los usuarios del OPAC de una biblioteca universitaria. Madrid.

Pressman, R. y Maxim, B. (2015). Software Engineering: A Practitioner's Approach. Eighth Edition.

Russell, J. y Cohn, R. (2012). Sharpdevelop. España.

Stolfa, J., Stolfa, S., O'Connor, R., Messnarz, R. (2017). Systems, Software and Services Process Improvement: 24th European Conference, EuroSPI 2017, Ostrava, Czech Republic, September 6-8, 2017, Proceedings. España.

Technology Management and Innovation, 31. Recuperado el 2 de junio 2017 de: https://www.jotmi.org/index.php/GT/article/download/art20/364.

Thibaud, C. (2006). MySQL 5: instalación, implementación, administración, programación. Barcelona.

Torres, A. (2014). Preparación de pedidos. España. 